\title{
Microcontroller Based Reprogrammable Digital Door Lock Security System by Using Keypad \& GSM/CDMA Technology
}

\author{
Mohammad Amanullah \\ (Lecturer, Department of Computer Science and Engineering, International Islamic University Chittagong. \\ Bangladesh.
}

\begin{abstract}
Now a day's Security has been a prime concern in the home or office management. Digital door lock security system provides security and safety to house or office owners, belongings, assets from being damaged by external agent or undesired strangers. We have used a new technology, incoming number verification system which gives more protection for controlling \& security system. As Conventional security system does not use any password, there is a chance to hack or break the system. In this regard we used a desire mobile number without verification which doesn't allow the door to be opend.This system is composed of the microcontroller based by using matrix keypad \& GSM/CDMA network. The microcontroller based digital door lock security system is an access control system that allows only authorized persons to access restricted area. The password is stored in PROM so that we can change it any time. The system has a matrix keypad. When anyone enter the code in the matrix keypad, microcontroller verify the codes. If that code is correct the device will operate and the door will be open. But if someone enter wrong code a red signal will be shown which means that the entered code is wrong.GSM/CDMA module can be used to operate the device, when anyone make a call from his mobile the receiving device which is set in main circuitry will receive the call. If the call is from desired number then operate the device and the door will be unlocked. An IPS circuit can be used for giving backup in case of emergency when there is a power failure.
\end{abstract}

Keywords: DTMF encoder and decoder, Global System for Mobile Communication, Micro-Controller, Mobile phone, and Matrix Keypad.

\section{Introduction}

Tones generated from DTMF keypad can identify what unit we want to control, as well as which unique function we want to perform. A standard $4 * 4$ matrix keyboard can be used support either DP or DTMF modes.DTMF encoder technologies does not require any radiation or any laser beam, not harmful, no limitation of range. It can be used from any distance using a simple telephone line or mobile phone. The UM91214B is a single chip, silicon gate. It provides dialing pulse (DP). IC UM91214B is consists of telephone set which is present in the remote place (Be your workspace) signal are sent through this telephone or mobile. For GSM/CDMA based controlling system, press a button in the telephone set keypad, a connection is made that generates a resultant signal of two tones at the same time. These two tones are taken from row frequency and column frequency. The resultant frequency signal is called "Duel Tone Multiple Frequency "These tone are identical and Unique.MT-8870 operating function include a band split filter that separates the high and low tones of the received pair, and a digital decoder that verifies both the frequency and duration of the received tones before passing the resulting 4-bit code to the output bus. 


\section{System Architecture \& Operation:}

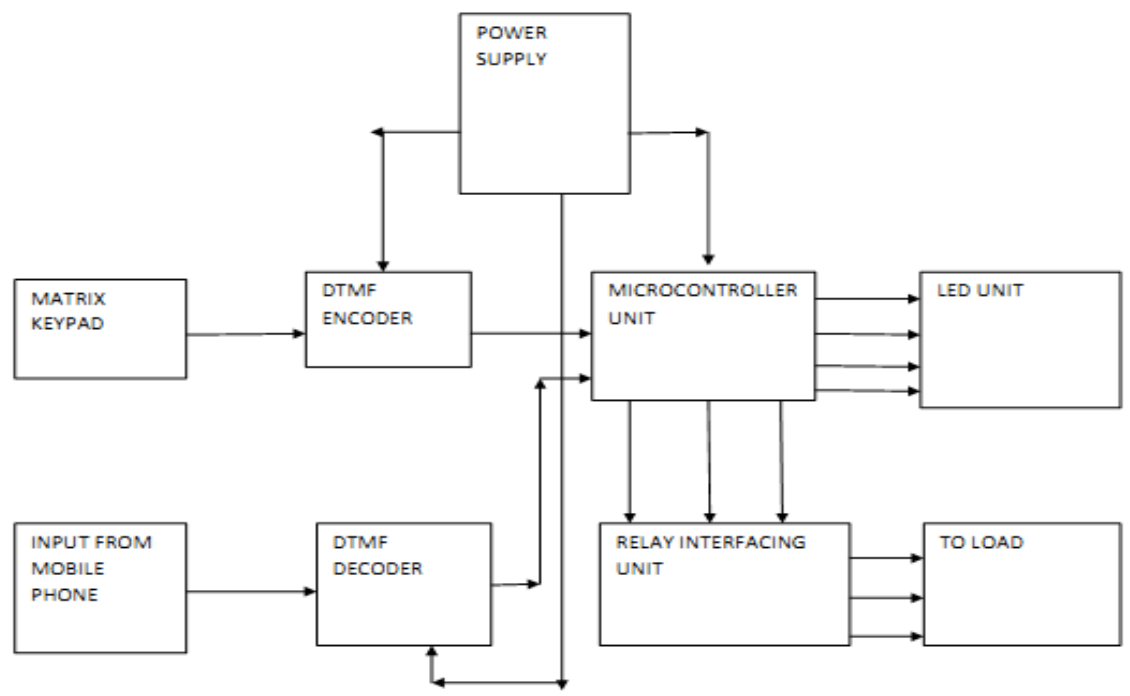

Fig 1. Shows the block diagram of the system.

In the above fig1.shows block diagram of the system keypad are a part of human machine interface where human interaction or human input is needed and play an important role in a small embedded system. A digital encoder is a device that converts tone signal into a sequence of digital pulse. By counting a single bit or by decoding a set of bits, the pulse can be converted to relative or absolute pulse can be converted to relative or absolute position measurement.DTMF decoder detects the dial tone from a cell phone and decodes the keypad pressed on the remote cell phone. Microcontroller units are used for special or fixed type operation. Microcontrollers are used in automatically controlled products and devices, such as remote control, appliances etc.Relay interfacing unit interface between the loads and controlling circuit. A relay is a protective device driven from controlling circuit directly.LED unit indicates the "ON" "OFF" position of the connected load. If LED is turn ON, then corresponding load is ON. When LED is OFF then corresponding load is OFF.DOOR have been used as LOAD.

\section{Design of System Hardware}

System hardware designing composed of ENCODER, DECODER and MICROCONTROLLER. These are consists of matrix keypad, IC-UM91214B, IC-(MT8870D), IC-(PIC-16F628A), Zener diode, LED, Resistor, Capacitor, Relay. Microcontroller verifies the codes. Encoder is a device that converts tone signal into sequence of digital pulse. Decoder detects the dial tone from a telephone line and decodes the keypad pressed on the remote telephone. Fig 2. Is the circuit diagram of digital door lock security system and Fig 3. Shows practical implementation. 


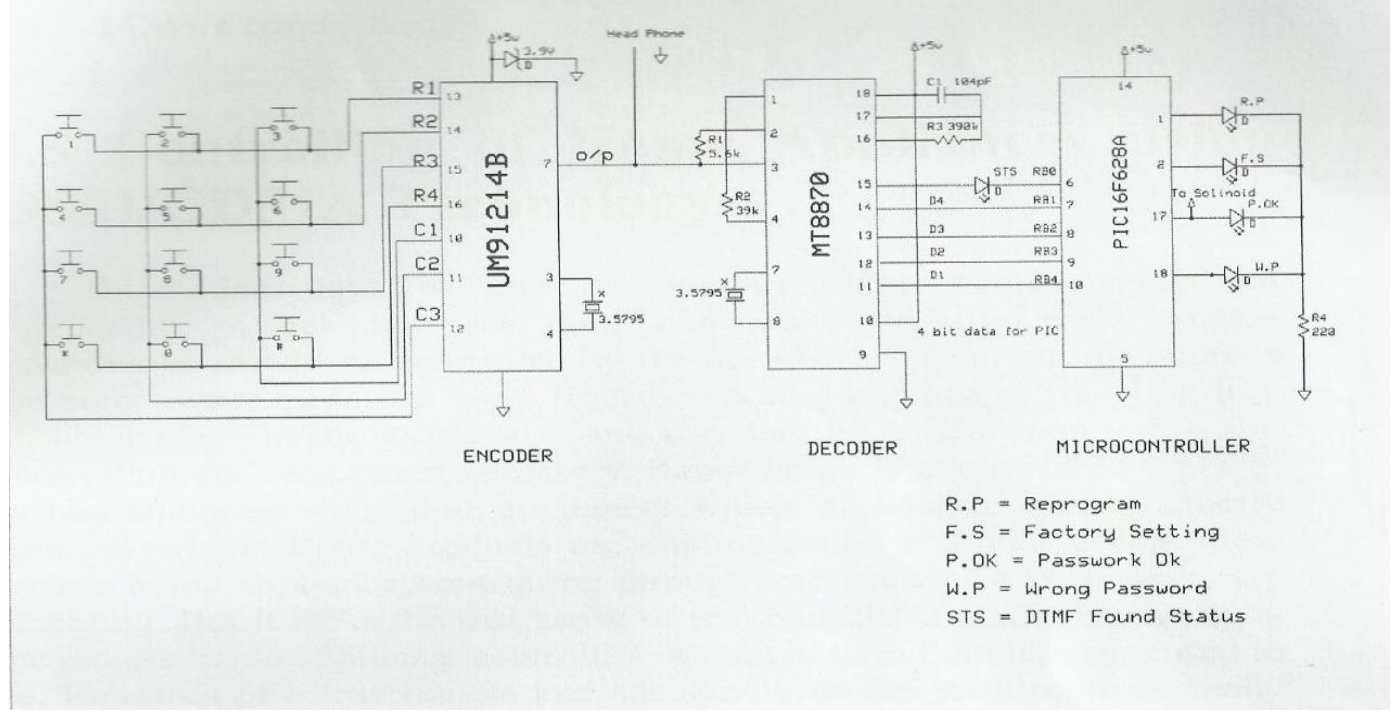

Fig 2. Circuit diagram of Digital Door lock security system.

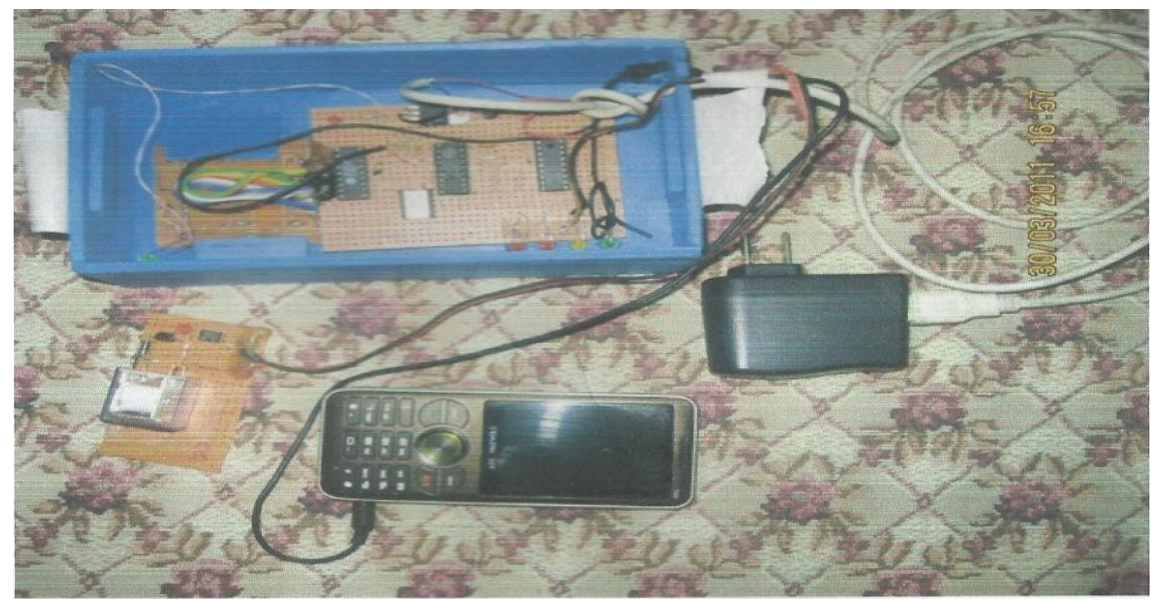

Fig 3. shows the practrical implementation of the circuit.

\section{Selection Of MICROCONTROLLER:}

Microcontroller is special types of processor which we used for special or fixed type operation. A Microcontroller is a small computer on a signal integrated circuit containing a processor core, memory and programmable input/output peripherals. The main function for PIC16F628A microcontroller which specify the $\mathrm{ON}$ and OFF state of the switches. The program for the microcontroller is designed in such a way that when we make a call from our transmitting device, it would be automatically received by Decoder circuit and it will offer a password code for security purpose. After verifying the password code it will give the access to operate the circuit.

\section{Working of IC Mt8870:}

The MT-8870 is a full DTMF receiver that integrates both band split filter and decoder function into a single 18-pin DIP. Its filter section uses switched capacitor technology for both the high and low group filters and for dial tone rejection. Its decoder uses digital counting techniques to detector and decodes all 16 DTMF tone pairs into a 4-bit code. External component count is minimized by provision of an on-chip differential input amplifier clock generator and latched tri-state interface bus.MT-8870 operating functions include a band split filter that separates the high and low tones of the received pair, and a digital decoder that verifies both the frequency and duration of the received tones before passing the resulting 4-bit code to the output bus. 


\section{Working of IC Um91214b:}

This unit consists of telephone set which is present in the remote place. This may be workspace (office/ school) phone or mobile phone or a phone in PCO. Signals are sent through this telephone. It uses DTMF encoder integrated circuit, chip UM91214B. This IC produces DTMF signals. It contains four row frequencies \& three column frequencies. The pins of the IC91214B from 12 to 14 produces high frequency column group and pins from 15 to 18 produces the low frequency row group. By pressing any key in the keyboard corresponding DTMF signal is available in its output pin at pin no 7.For producing the appropriate signals it is necessary that a crystal oscillator of $3.58 \mathrm{MHz}$ is connected across its pins $3 \& 4$ so that it makes a part of its internal oscillator. This encoder IC requires a voltage of $3 \mathrm{~V}$.For that IC is wired around 4.5 battery and $3 \mathrm{~V}$ backup $\mathrm{V}_{\mathrm{CC}}$ for this IC is supplied by using $3.2 \mathrm{~V}$ zener diode. By pressing the number 5 in the keypad the output tone is produced which is the resulting of addition of two frequencies at pin no $13 \& 16$ of the IC and respective tone which represents number ' 5 ' in keypad is produced at pin no 7 of the IC (This signal is sent to the local control system through telephone line via exchange).

\section{Keypad Matrix:}

Password is given in our system by using keypad. Keypad may be similar as telephone set's keypad. Function of this keypad is also same as the telephone. When you press buttons on the keypad, a connection is made that generates two tones at the same time. A "Row" tone and a "column" tone. These two tones identify the key you pressed to any equipment you are controlling. If the keypad is on your phone, the telephone company's "central office "equipment knows what number you are dialing by these tones, and will switch your call accordingly. If you are using a DTMF keypad to remotely control equipment, the tones can identify what unit you want to control, as well as which unique function you want it to perform. When you press the digit 1 on the keypad, you generate the tones $1209 \mathrm{~Hz}$ and $697 \mathrm{~Hz}$. Pressing the digit 2 will generate the tones $1336 \mathrm{~Hz}$ and $697 \mathrm{~Hz}$. Sure, the tone 697 is the same for both digits, but it takes two tones to make a digit and the decoding equipment knows the difference between the $1209 \mathrm{~Hz}$ that would complete the digit 1 , and a1336 Hz that completes a digit 2 .There are many methods but the basic logic is same .

\section{GSM/CDMA BASED CONTROLLING SYSTEM}

If you press a button in the telephone set keypad, a connection is made that generates a resultant signal of two tones at the same time. These two tones are taken from a row frequency and a column frequency. The resultant frequency signal is called "DTMF". In GSM, DTMF signal is discretized.These tones are identical and unique. A DTMF signal is the algebraic sum of two different audio frequencies, and can be expressed as follows:

$\mathrm{F}(\mathrm{t})=\mathrm{A}_{o} \operatorname{Sin}\left(2 * \Pi * \mathrm{f}_{\mathrm{a}} * \mathrm{f}_{\mathrm{b}} * \mathrm{t}\right)+\mathrm{B}_{0} \operatorname{Sin}\left(2 * \prod * \mathrm{f}_{\mathrm{a}} * \mathrm{f}_{\mathrm{b}} * \mathrm{t}\right) \ldots \ldots \ldots .(1)$

Where $f_{a}$ and $f_{b}$ are two different frequencies $A$ and $B$ as their peak amplitudes. Each of the low and high frequency groups comprise four frequencies from the various keys present on the telephone keypad. Two different frequencies, one from the high frequency group and another from the low frequency group are used to produce a DTMF signal to represent the pressed key. The amplitude of the two sine waves should be such that $(0.7<(\mathrm{A} / \mathrm{B})<0.9) \mathrm{V}$

The frequencies are chosen such that they are not the harmonics of each other. The frequencies associated with various keys on the keypad. When you send these DTMF signals to the telephone exchange through cables, the servers in the telephone exchange identifies these signals and makes the connection to the person you are calling.

\section{FILTER SECTION}

Separation of the low-group and high group tones is achieved by applying he DTMF signal to the inputs to two sixth-order switched capacitor band pass filters, the bandwidths of which correspond to the low and high group frequencies. The filter section also incorporates notches at 350 and $440 \mathrm{~Hz}$ for exceptional dial tone rejection.

Each filter output is followed by a single order switched capacitor filter section which smoothes the signal prior to limiting. Limiting is performed by high-gain comparators which are provided with hysteresis to prevent detection of unwanted low-level signals. The outputs of the comparators provide fill rail logic swings at the frequencies of the incoming DTMF signals.

The filter also incorporates notches at 350 and $440 \mathrm{~Hz}$, providing excellent dial tone rejection. Each filter output is followed by a single-order switched capacitor section that smoothes the signals prior to limiting. Signal limiting is performed by high gain comparators provided with hysteresis to prevent detection of unwanted low-level signals and noise. The MT- 8870 decoder uses a digital counting technique to determine the frequencies of the limited tones and to verify that they correspond to standard DTMF frequencies. When the detector recognizes the simultaneous presence of two valid tones (known as signal condition), it raises the Early 
Steering flag(ESt). Any subsequent loss of signal condition will cause ESt to fall. Before a decoded tone pair is registered, the receiver checks for valid signal duration( referred to as character recognition-condition) this check is performed by an external RC time constant driven by ESt. a short delay to allow the output latch to settle, the delayed steering output flag( $\mathrm{StD})$ foes high, signaling that a received tone pair has been registered. The contents of the output latch are made available of the 4-bit output bus by raising the three state control input $(\mathrm{OE})$ to logic high. Inhibit mode is enabled by a logic high input to pin 5(INH). It inhibits the detection of $1633 \mathrm{~Hz}$.

The output code will remain the same as the previous detected code. On the MT- 8870 models, this pin is tied to ground (logic low)

The input arrangement of the MT-8870 provides a differential input operational amplifier as well as a bias source (VREF) to bias the inputs at mid-rail. Provision is made for connection of a feedback resistor to the opamp output (GS) for gain adjustment.

\section{GSM/CDMA Network based OPERATION:}

Digital Door Lock security system can be operated in two ways. One is by using matrix keypad and another one is by using GSM/CDMA network. A DTMF circuit is employed which is connected to a mobile phone as a receiving device. For different keys of the mobile or telephone DTMF decoder decodes multiple frequencies which will be further used to control the household or Industrial Appliance. A program is written for PIC16F628A microcontroller which specify the ON and OFF state of the switches. The microcontroller program is loaded in the PIC16F628A microcontroller using a loader. An IPS circuit can be used for giving back up in case of power failure.

And our mobile or telephone is the transmitting device of this experiment. The program for the microcontroller is so designed that when we make a call from our transmitting device, it would be received by decoder circuit and it will offer a pass code barrier for security purposes. After verifying the pass code it will give the access to operate the circuit.

\section{Conclusion And Further Recommendations}

This paper presents one solution for user friendly, easily maintained, lower power consumption, less expensive remote door lock system. The system is based on matrix keypad and GSM/CDMA technology. In this project we have used a new technology incoming number verification system for controlling and security system. We used a desired mobile number without verification which does not allow the door to be opened. Some technologies are commercially available which allow remote home appliance controlling through internet which is undoubtedly emerging. But it lacks the true sense of real mobility. In search of a true remote and adequately secure solution to be really effective and realizable, mobile telephony is better than any other solutions. Of late, the system can also be the combination of hardware and software and Artificial intelligence based Door lock security system design on the future development needs. The system also can be used in hospitals, libraries, parks and museums, such as urgent security and remote observation of the site for the protection purpose from threat of damage and destruction.

\section{References}

[1] Syam Krishna, J. Ravindra, Design and Implementation of Remote Home Security System Based on WSNS and GSM Technology, IJESAT, Vol. 2, Special issue-1,PP.139-142, January-February, 2012.

[2] Design and Implementation of Pyroelectric Infrared Sensor Based Security System Using Microcontroller, Proceeding of the 2011 IEEE Students' Technology Symposium 14-16 January, 2011, IIT Kharagpur.

[3] Automated advanced industrial and home security using GSM and FPGA, Department of Electronics and Communication Engineering BV.CEngineering college, Odalarevu, Amalapuram,India.

[4] AT Commands Set for Nokia GSM and WCDMA products, version 1.2, July 2005, available

[5] Ren Fengyuan, Huang Haining, Lin Chuang, "Wireless sensor network", Journal of Software Vol. 14, No. 7, PP.1282-1291, March, 2003 (in Chinese).

[6] http://www.scribd.com/doc/27053839/activating-an-electrical-device-via-dtmf-telephone

[7] http://www.quasarelectronics.com/3075-pic-microcontroller-traine-with-5-function.htm

[8] http://www.electro-tech-online.com/microcontrollers/18814-dtmf-tone-pic16f84.html

[9] http://www.embedds.com/-software-based-dtmf-remote-control

[10] http://www.electronics-lab.com/blog/?=microcontroller\&paged $=43$

[11] http://electrofriends.com/projects/basis-electronics/device-control-using-telephone 